\title{
Colonic Chicken Skin Mucosa Surrounding Colon Polyps Is an Endoscopic Predictive Marker for Colonic Neoplastic Polyps
}

\author{
Yu Mi Lee ${ }^{1,2}$, Kyung Ho Song ${ }^{3}$, Hoon Sup Koo², Choong-Sik Lee ${ }^{4}$, Inseok Ko ${ }^{5}$, Sang Hyuk Lee², and Kyu Chan Huh² \\ ${ }^{1}$ Health Screening and Promotion Center, Konyang University Hospital, ${ }^{2}$ Department of Gastroenterology, College of Medicine, \\ Konyang University, Daejeon, ${ }^{3}$ Department of Gastroenterology, CHA University Ilsan Medical Center, Ilsan, Departments of ${ }^{4}$ Pathology \\ and ${ }^{5}$ Biomedical Informatics, College of Medicine, Konyang University, Daejeon, Korea
}

\section{Article Info}

Received June 24, 2021

Revised September 13, 2021

Accepted October 21, 2021

Published online January 7, 2022

\section{Corresponding Author}

Kyu Chan Huh

ORCID https://orcid.org/0000-0003-3746-8419

E-mail kchuh2020@daum.net

Yu Mi Lee and Kyung Ho Song contributed equally to this work as first authors.

\begin{abstract}
Background/Aims: Narrow band imaging provides an accurate diagnosis of colonic polyps. However, these diagnostic modalities are not used as standard endoscopic tools in most institutions. This study aims to investigate whether the chicken skin mucosa (CSM) surrounding the colon polyp yields additional information about colorectal polyps, including histological differentiation of neoplastic and non-neoplastic polyps, under conventional white light colonoscopy.

Methods: This study prospectively observed 173 patients who underwent endoscopic polypectomy and reviewed the clinical data and pathologic reports of 313 polyps from a university hospital. Two endoscopists each performed colonoscopy and polypectomy and assessed the CSM. The association between CSM surrounding colorectal polyps and histology was analyzed. Results: The majority (91.3\%) of CSM-positive polyps were neoplastic (sensitivity, $37.90 \%$; specificity, $86.15 \%$; $\mathrm{p}<0.001$ ). In logistic regression, the neoplastic polyps were associated with positive CSM (adjusted odds ratio [OR], 3.51; 95\% confidence interval [Cl], 1.45 to 9.25; $\mathrm{p}=0.007$ ), protruded polyps (adjusted OR, 4.85; $95 \% \mathrm{Cl}, 1.65$ to $17.23 ; \mathrm{p}=0.008$ ), and neoplastic histology-associated pit pattern (pit III, IV, and V) (adjusted OR, 10.14; 95\% Cl, 4.85 to 22.12; $\mathrm{p}=0.000$ ). Furthermore, advanced adenomas were associated with positive CSM (adjusted OR, 5.64; $95 \% \mathrm{Cl}, 1.77$ to 20.28; $p=0.005$ ), protruded polyps (adjusted OR, 3.30; 95\% Cl, 1.15 to 9.74; $p=0.026$ ), and $\geq 10 \mathrm{~cm}$ polyp size (adjusted OR, 18.56; $95 \% \mathrm{Cl}, 3.89$ to 147.01; $p=0.001$ ).

Conclusions: Neoplastic and advanced polyps were associated with CSM-positive polyps. These findings suggest that CSM is a useful marker in differentiating neoplastic polyps and advanced polyps under conventional white colonoscopy. (Gut Liver 2022;16:754-763)
\end{abstract}

Key Words: Colon neoplasms; Macrophages; Carcinogenesis; Colonoscopy

\section{INTRODUCTION}

Colorectal cancer (CRC) is the third most commonly diagnosed cancer in males, the second most frequently detected malignancy in females, and ranked third in terms of mortality rate in Korea in $2019 .{ }^{1}$ Colon polypectomy and continuous surveillance via colonoscopy are important for reducing the incidence and mortality of CRC.,3

The clinical significance of neoplastic polyps differs from that of non-neoplastic polyps. According to the theory of adenoma-carcinoma sequence, ${ }^{4}$ accurate differentiation from non-neoplastic polyps and clear polypec- tomy is necessary because neoplastic polyps can progress to CRC. Removal of non-neoplastic polyps not only entails pathological investigations and procedural costs, ${ }^{5}$ but also increases complications. In addition, non-neoplastic polypectomy does not reduce the risk of CRC. Therefore, it is important to differentiate non-neoplastic from neoplastic lesions before performing colon polypectomy.

Various methods have been attempted for accurate diagnosis and removal of precancerous lesions (neoplastic polyps), such as narrow band imaging (NBI), endocytoscopy, and laser scanning confocal microscopy. ${ }^{6-8}$ A study by Sano et al. ${ }^{9}$ found that magnifying colonoscopy with 
NBI had high sensitivity, specificity, and accuracy (96.4\%, $92.3 \%$, and $95.3 \%$, respectively) in differentiating neoplastic from non-neoplastic lesions. However, NBI combined with magnifying colonoscopy is not clinically used as a standard endoscopic intervention in most institutions.

Specific endoscopic finding in the widely used conventional white light colonoscopy is suggested to differentiate neoplastic polyps. Chicken skin mucosa (CSM) was first described based on specific morphological changes surrounding a colorectal adenoma found in conventional colonoscopy in $1998 .{ }^{10}$ CSM was superficially visible as yellow-speckled colonic mucosa. The histopathology of CSM revealed lipid-filled macrophages in the lamina propria. The authors found no CSM without an associated neoplasm, and this change was more frequently detected in association with neoplasms of the distal colon than in the proximal colon. Conversely, Nowicki et al. ${ }^{11}$ suggested that CSM with childhood polyps is not a preneoplastic lesion. In this study, CSM was associated with juvenile polyps (both solitary and juvenile polyposis coli) that are nonmalignant.

However, recent studies show that CSM surrounding an adult colorectal polyp is associated with advanced colorectal adenoma via gene expression. ${ }^{12}$ In addition, a Korean study showed that CSM was associated with advanced pathology, including villous adenoma and high-grade dysplasia. ${ }^{13}$ This study aimed to find out whether CSM is associated with neoplastic polyps. It is important to determine whether the polyp is neoplastic before polypectomy to determine the removal and extent of ablation. Therefore, this study checked whether CSM could be used as an endoscopic marker in conventional white colonoscopy, such as a vascular pattern on NBI with chromoendoscopy.

The researchers investigated whether the CSM surrounding the colon polyp yielded additional information about colorectal polyps under conventional white light colonoscopy, including advanced histology and differentiation of neoplastic from non-neoplastic polyps.

\section{MATERIALS AND METHODS}

\section{Patients}

Men and women aged between 18 and 85 years who underwent screening, surveillance, or diagnostic colonoscopy were enrolled in this study. The exclusion criteria covered patients who could not undergo polypectomy or those with polyps removed by forceps biopsy, and patients with poor bowel preparation or with a prior history of CRC or inflammatory bowel disease. A total of 422 patients satisfied the inclusion criteria and underwent colonoscopy.
Among them, 173 patients underwent polypectomy at least once. The researchers prospectively evaluated 173 patients who underwent endoscopic mucosal resection (EMR) of colonic polyps following a screening colonoscopy. The clinical data and pathologic reports of 313 polyps were reviewed at a university hospital between June and December 2013 (Fig. 1). All patients who underwent colonoscopy received written details of the medical records program and a standard questionnaire asking about their past medical history, current medications, and family history. The study protocol was approved by the Institutional Review Board of Konyang University Hospital (IRB number: 201305-003). Informed consent was obtained.

\section{Colonoscopy and EMR}

All examinations were performed by two experienced colonoscopists using high-definition colonoscopy (CF Q260AI, CF Q260AL, and CV 260; Olympus, Tokyo, Japan). During colonoscopy, the location and number of polyps were recorded, and $\geq 10$ colon landmarks (terminal ileum, ileocecal valve, cecum, ascending colon, hepatic flexure, transverse colon, splenic flexure, descending colon, sigmoid colon, and rectum) were imaged. The pit pattern of the polyp was closely observed under conventional white light colonoscopy. CSM was diagnosed by an expert colonoscopist who conducted the examination.

A retrospective study was conducted on the subject of this study. ${ }^{14}$ The kappa value for inter-observer agreement was $0.555(\mathrm{p}<0.001)$. The two independent endoscopists who participated in a previous study conducted colonoscopy in this study. The polyp size was measured at the time of biopsy.

EMR was performed for polyps measuring $>5 \mathrm{~mm}$. Polyps that underwent EMR received a submucosal injection

600 Patients enrolled in prospective clinical trials

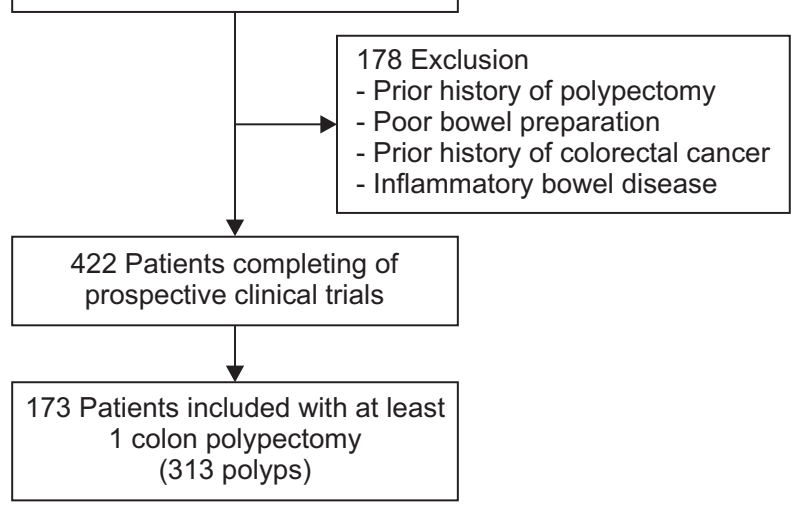

Fig. 1. Flowchart of the patients. 
of $0.9 \%$ saline solution mixed with 1:100,000 epinephrine and indigo carmine. Following submucosal injection, yellowish spots around the polyp were occasionally observed. Afterwards, the polyp was resected using a snare. Cold snaring was performed sometimes, so the polyps were removed after injection. All resected polyps were reviewed and confirmed by expert pathologists according to the Vienna classification. ${ }^{15}$

\section{Definition of terms used in this study}

CSM was classified into three based on their characteristics. Type 1 CSM was confirmed before injection, type 2 CSM was observed after injection, and type 3 CSM was not observed (Fig. 2). Types 1 and 2 were included as positive CSM findings. The colonic polyps were divided based on location; those in the proximal colon (cecum, ascending colon, hepatic flexure, and transverse colon) and the distal colon (splenic flexure, descending colon, sigmoid colon, and rectum) ${ }^{16,17}$ Neoplastic polyps are defined as polyps with epithelial dysplasia, which included adenomas and carcinomas. ${ }^{18}$ Although serrated adenoma is associated with hyperplastic polyps, it also has malignant potential. Thus, it was considered a neoplastic polyp. Advanced pathology was indicated by a colonic adenoma with $>25 \%$ villous structure, high-grade dysplasia, and carcinoma in situ. ${ }^{13,19}$ The Paris classification was used to describe polyp morphology. The sessile (0-Is), slightly elevated (0-IIa), flat (0-IIb), and laterally spreading tumor (LST) were defined as flat lesions, and pedunculated (0-Ip) and sub-pedunculated (O-Isp) tumors were defined as protruding lesions. ${ }^{20}$
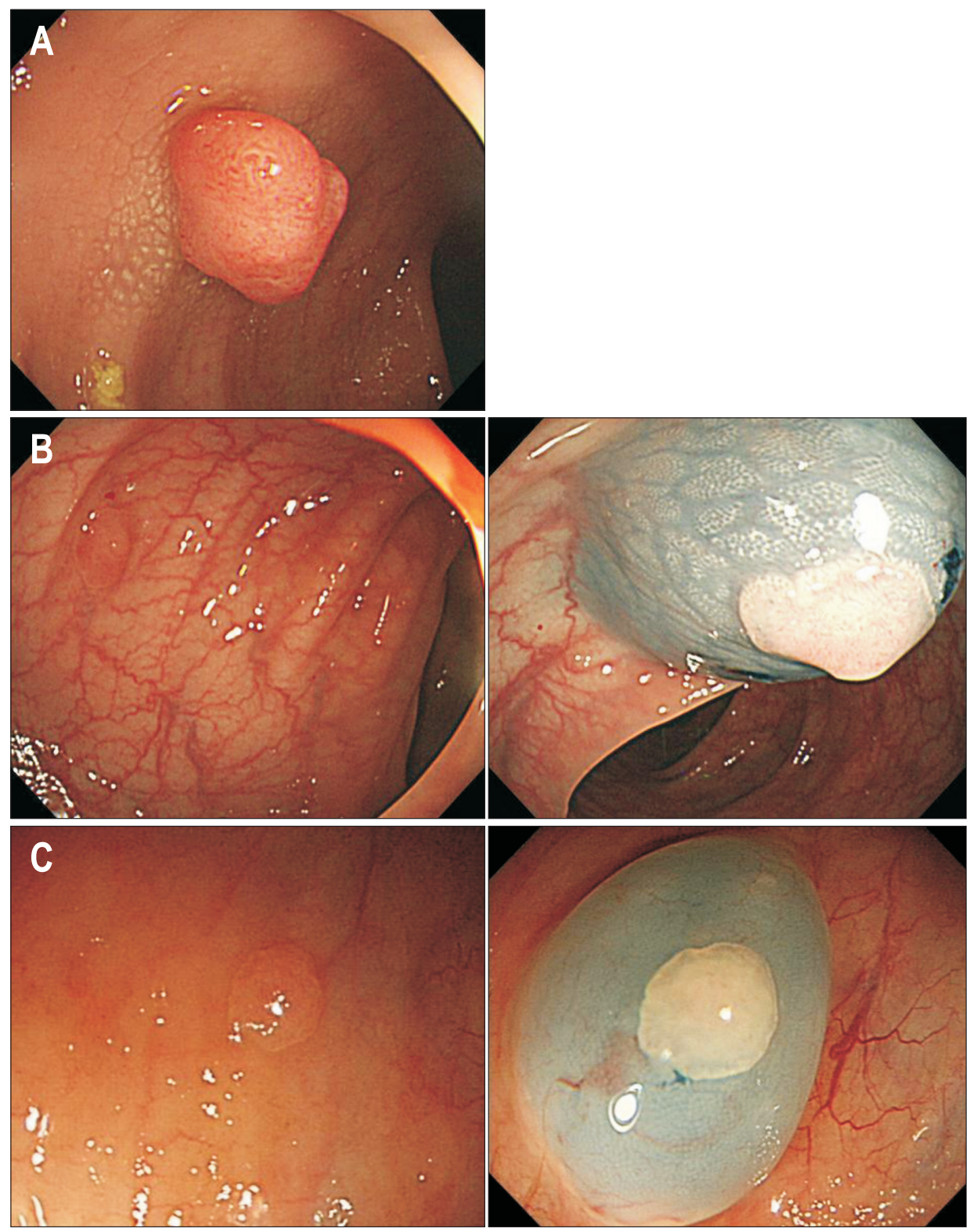

Fig. 2. Endoscopic appearances of chicken skin mucosa (CSM). (A) Type 1 CSM (confirmed before injection). (B) Type 2 CSM (confirmed after injection). (C) Type 3 CSM (not observed). 
The pit pattern was classified as described by Kudo et al., ${ }^{21}$ ranging from type I to type V. Types I and II represent non-neoplastic lesions, whereas types IIIL, IIIS, IV, and V are neoplastic lesions.

\section{Statistical analysis}

All analyses were performed using the R software (version 3.5.3) from the R Foundation for Statistical Computing (Vienna, Austria) and IBM SPSS version 22.0 (IBM Corp., Armonk, NY, USA). The relationship between CSM, clinical factors, and the characteristics of colonic adenomas was analyzed using the Fisher exact test, whereas continuous variables were analyzed using the Student ttest. Logistic regression was used to examine the relationship between neoplastic polyps and CSM presence. Two dependent variables, neoplastic polyps and advanced adenomas, were used in this study. The data regarding neoplastic polyps $(n=313)$ and advanced adenomas $(n=244)$ were used to exclude missing values for each dependent variable. Statistical significance was set at $\mathrm{p}<0.05$. For posthoc sample size calculation, logistic regression with the $\mathrm{z}$ test was applied with $\alpha(0.05)$, power $(0.8,0.9,0.95)$ using $\mathrm{G}^{*}$ Power software for windows (version 3.1.9.4). ${ }^{22}$

\section{RESULTS}

\section{Baseline characteristics of population}

This study included 173 patients who underwent EMR following screening colonoscopy. The mean age of the patients was $59.4 \pm 11.7$ years, and 111 patients (64.2\%) were male (Table 1). No distinguishing clinical characteristics were found between the CSM-positive and CSMnegative groups. Pathologically, CSM is characterized by aggregates of lipid-laden macrophages, suggesting that the body mass index or lipid status of patients is associated with CSM. However, the mean body mass index of the patients was $24.20 \pm 2.97$, that of the CSM-positive group was $24.44 \pm 2.87$, and that of the CSM-negative group was $23.74 \pm 3.13$, with no significant differences between the two groups. In addition, there was no association between hypertension, dyslipidemia, and CSM.

\section{Histopathological appearance of CSM}

All cases of CSM were confirmed endoscopically; however, only some cases included at the time of EMR were confirmed histologically. Many lipid-laden macrophages were observed in the superficial colorectal mucosa in cases of CSM with tubular adenoma and CSM with carcinoma in situ (Fig. 3). In cases where CSM was observed after injection, an increase in foamy macrophages in the lamina propria was noted.

\section{Characteristics of polyps related to CSM}

A total of 173 patients underwent EMR following screening colonoscopy, and EMR was performed on 313 polyps. Of the polyps with EMR, 70.0\% were tubular adenomas $(n=219)$, and $9.6 \%$ showed advanced histology $(n=30)$. CSM was observed in 103 of the 313 polyps (32.9\%). Among 103 CSM-positive polyps, 94 (91.3\%) were neoplastic polyps and 56 out of 210 CSM-negative polyps (26.7\%) were non-neoplastic. The prevalence of neoplastic polyps was significantly higher in polyps with CSM than those without CSM ( $91.3 \%$ vs $73.3 \%$; $\mathrm{p}<0.001$ ). In addition, advanced histology was more frequently observed in polyps accompanied by CSM, than in those that were not associated with CSM (23.2\% vs $5.2 \%$, respectively, $\mathrm{p}<0.001)$.

The polyps with CSM were mainly distributed in the distal colon (proximal $16.5 \%$ vs distal $83.5 \%$ ). The median polyp size was $7.06 \pm 4.85 \mathrm{~mm}$. A polyp without CSM had a median size of $6.30 \pm 3.55 \mathrm{~mm}$, whereas the polyp with CSM measured $8.63 \pm 6.52 \mathrm{~mm}$, suggesting significant difference ( $\mathrm{p}=0.001)$. In polyps showing pits III, IV, and V, the proportion of polyps with CSM and without CSM were $91.3 \%$ and $77.6 \%$, respectively $(\mathrm{p}=0.003)$ (Table 2$)$.

Table 1. Baseline Characteristics of the Subjects

\begin{tabular}{|c|c|c|c|c|}
\hline Variable & Total ( $n=173)$ & Presence of CSM (n=51) & Absence of CSM ( $n=122)$ & $\mathrm{p}$-value \\
\hline Age, yr & $59.35 \pm 11.74$ & $59.24 \pm 11.75$ & $59.39 \pm 11.78$ & 0.936 \\
\hline Male sex & $111(64.2)$ & $28(54.9)$ & $83(68.0)$ & 0.101 \\
\hline $\mathrm{BMI}, \mathrm{kg} / \mathrm{m}^{2}$ & $24.20 \pm 2.97$ & $23.74 \pm 3.13$ & $24.44 \pm 2.87$ & 0.141 \\
\hline Hypertension & $63(36.4)$ & 20 (39.2) & 43 (35.2) & 0.621 \\
\hline Diabetes mellitus & $33(19.1)$ & $5(9.8)$ & $28(23.0)$ & 0.045 \\
\hline Dyslipidemia & $18(10.4)$ & $3(5.9)$ & 15 (12.3) & 0.208 \\
\hline Smoking & $46(26.6)$ & $9(17.6)$ & $37(30.3)$ & 0.129 \\
\hline Consumption of alcohol & 30 (17.3) & $8(15.7)$ & $22(18.0)$ & 0.710 \\
\hline Family history of colorectal cancer & $8(4.6)$ & $4(7.8)$ & 4 (3.3) & 0.192 \\
\hline
\end{tabular}

Data are presented as the mean \pm SD or number $(\%)$.

CSM, chicken skin mucosa; BMI, body mass index. 

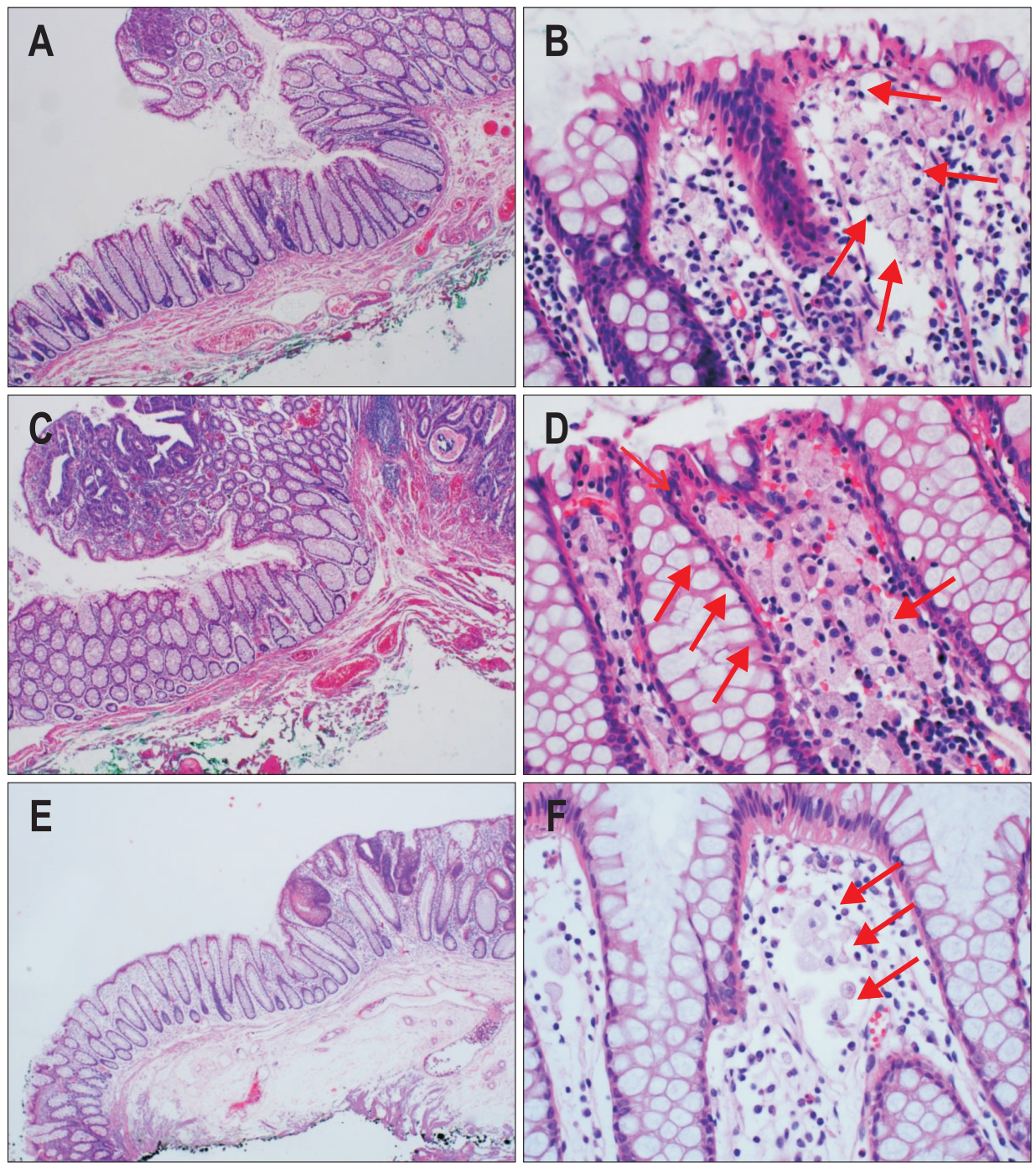

Fig. 3. Histological appearances of chicken skin mucosa (CSM). (A) Histological appearance of tubular adenoma and the surrounding mucosa (H\&E, ×40). (B) Histological appearance of CSM, including lipidladen macrophages (arrows) in the lamina propria $(H \& E, \times 400)$. (C, D) Histological appearance of carcinoma in situ and the surrounding mucosa $(H \& E, \times 40)$ and CSM, including lipidladen macrophages (arrows) in the lamina propria. (E, F) Histological appearance of tubular adenoma and the surrounding mucosa, which is observed showing CSM after injection for endoscopic mucosal resection and CSM, including lipid-laden macrophages (arrows) in the lamina propria $(H \& E, \times 400)$.

CSMs were divided into two types: those that were present before and after injection. A total of 76 of 84 (90.5\%) type 1 CSM, 18 out of 19 (94.7\%) type 2 CSM, and 154 out of $210(73.3 \%)$ type 0 CSM were neoplastic polyps, showing significant differences in the proportion of neoplastic polyps $(\mathrm{p}=0.001)$. A similar pattern was observed in advanced histology $(24.7 \%$ vs $16.7 \%$ vs $5.2 \%$, $\mathrm{p}<0.001)$ (Supplementary Table 1).

Based on the pit pattern observed under conventional colonoscopy, 225 cases (90.7\%) showed pit pattern types III, IV, and V among neoplastic polyps, and 33 cases (50.8\%) showed pit I and II types among non-neoplastic polyps $(\mathrm{p}<0.001)$. The polyps in pits III, IV, and V were more common in the flat type (flat vs protruding, $74.3 \%$ vs 25.7\%; $\mathrm{p}=0.016$ ) (Supplementary Table 2).

The diagnostic performance of CSM and the pit pattern of neoplastic polyps were compared. CSM had a sensitivity of $37.90 \%$ and specificity of $86.15 \%$, and the neoplastic pit pattern showed a sensitivity of $90.73 \%$ and specific- ity of $50.77 \%$. Diagnosis of neoplastic polyps using both CSM and pit patterns showed a sensitivity of $35.08 \%$ and specificity of $89.23 \%$. Additionally, diagnostic performance using either CSM or pit pattern showed a sensitivity of $93.55 \%$ and specificity of $47.69 \%$ (Table 3 ).

\section{Multiple logistic regression analyses of neoplastic polyps and advanced adenomas}

Two dependent variables, neoplastic polyp and advanced adenoma, were analyzed. First, univariate and multivariate logistic regression analyses were performed with several independent variables expected to affect the dependent variable, neoplastic polyps. The neoplastic polyps were associated with positive CSM (adjusted odds ratio [OR], 3.51; 95\% confidence interval [CI], 1.45 to 9.25; $\mathrm{p}=0.007$ ), protruding polyps (adjusted $\mathrm{OR}, 4.85 ; 95 \%$ CI, 1.65 to 17.23 ; $\mathrm{p}=0.008$ ), neoplastic pit pattern (pits III, IV, and V) (adjusted OR, 10.14; 95\% CI, 4.85 to 22.12; $\mathrm{p}=0.000$ ), and hypertension (adjusted OR, 2.99; 95\% CI, 
Table 2. Characteristics of Polyps with or without CSM

\begin{tabular}{|c|c|c|c|c|}
\hline Variable & Total & Presence of CSM* & Absence of CSM & $\mathrm{p}$-value \\
\hline Histology I & 313 & 103 & 210 & $<0.001$ \\
\hline Neoplastic & & 94 (91.3) & 154 (73.3) & \\
\hline Non-neoplastic & & $9(8.7)$ & $56(26.7)$ & \\
\hline Histology II & 249 & 95 & 154 & $<0.001$ \\
\hline Advanced histology & & $22(23.2)$ & $8(5.2)$ & \\
\hline Tubular adenoma & & 73 (76.8) & $146(94.8)$ & \\
\hline Morphology* & 313 & 103 & 210 & 0.022 \\
\hline Flat (Illa, Is, LST) & & $71(68.9)$ & $170(81.0)$ & \\
\hline Protruded (Isp, Ip) & & $32(31.1)$ & 40 (19.0) & \\
\hline Location & 313 & 103 & 210 & $<0.001$ \\
\hline Proximal colon & & $17(16.5)$ & 112 (53.3) & \\
\hline Distal colon & & 86 (83.5) & $98(46.7)$ & \\
\hline Size, mm & 313 & 103 & 210 & 0.001 \\
\hline$<5$ & & $19(18.4)$ & 68 (32.4) & \\
\hline 5 to $<10$ & & $52(50.5)$ & $110(52.4)$ & \\
\hline$\geq 10$ & & $32(31.1)$ & $32(15.2)$ & \\
\hline Size of polyps, mm & $7.06 \pm 4.85(2-50)$ & $8.63 \pm 6.52(2-50)$ & $6.30 \pm 3.55(2-30)$ & 0.001 \\
\hline Pit pattern & 313 & 103 & 210 & 0.003 \\
\hline Pit I, II (non-neoplastic) & & $9(8.7)$ & $47(22.4)$ & \\
\hline Pit III, IV, V (neoplastic) & & 94 (91.3) & $163(77.6)$ & \\
\hline
\end{tabular}

Data are presented as the number (\%) or median \pm SD (range).

CSM, chicken skin mucosa; LST, laterally spreading tumor.

*The sessile (0-Is), slightly elevated (0-Ila), flat (0-IIb), and LST were defined as flat lesions, and pedunculated (0-Ip) and sub-pedunculated (0-Isp) tumors were defined as protruding lesions.

Table 3. Neoplastic Polyp-Diagnostic Performance of Chicken Skin Mucosa (CSM) and Pit Pattern

\begin{tabular}{lcccc}
\multicolumn{1}{c}{ Variable } & Sensitivity, \% & Specificity, \% & $\begin{array}{c}\text { Positive predictive } \\
\text { value, \% }\end{array}$ & $\begin{array}{c}\text { Negative predictive } \\
\text { value, \% }\end{array}$ \\
\hline CSM positive & 37.90 & 86.15 & 91.26 & 26.67 \\
Neoplastic pit pattern & 90.73 & 50.77 & 87.55 & 58.93 \\
CSM positive or neoplastic pit pattern & 93.55 & 47.69 & 87.22 & 65.96 \\
CSM positive and neoplastic pit pattern & 35.08 & 89.23 & 92.55 & 26.48 \\
\hline
\end{tabular}

\subsection{9 to $7.28 ; \mathrm{p}=0.013)$.}

The independent variable advanced adenoma was associated with positive CSM (adjusted OR, 5.64; 95\% CI, 1.77 to $20.28 ; \mathrm{p}=0.005$ ), protruding polyps (adjusted $\mathrm{OR}$, 3.30 ; $95 \% \mathrm{CI}, 1.15$ to $9.74 ; \mathrm{p}=0.026$ ), and a polyp size $\geq 10$ $\mathrm{cm}$ (adjusted OR, 18.56; 95\% CI, 3.89 to $147.01 ; \mathrm{p}=0.001$ ) (Table 4).

\section{DISCUSSION}

CSM is a mucosal abnormality defined by a yellowspeckled pattern in the colon seen under conventional light endoscopy. CSM is similar to xanthoma, which is a large number of lipid-laden macrophages aggregated in the mucosa. The clinical significance of CSM remains unclear. According to El-Hodhod et al., ${ }^{23}$ it is regarded as a benign compensatory response evoked by the mechanical effect of the polyp. Based on a study involving 36 patients with CSM among 240 patients with juvenile polyps, most of the CSM with the unique thickening of the muscularis mucosa, especially around larger polyps, disappeared after polypectomy. In addition, Nowicki et al. ${ }^{24}$ reported that CSM is a common finding in children with juvenile polyps, which have limited malignant potential, probably as a result of local mucosal trauma rather than a preneoplastic lesion.

However, in the present study, CSM was demonstrated as one of the factors associated with neoplastic polyps. The multivariate logistic regression analysis revealed an association with neoplastic polyp, and the adjusted OR was 3.51 when the CSM was positive. Furthermore, the association with the polyp showing advanced histology revealed a statistically significant adjusted OR of 5.14 for positive CSM. These findings suggest that CSM is an independent endosocopic predictor of neoplastic polyps and advanced 
Table 4. Simple and Multiple Logistic Regression Analyses for Neoplastic Polyps and Advanced Adenoma

\begin{tabular}{|c|c|c|c|c|c|c|c|c|}
\hline \multirow[b]{2}{*}{ Variable } & \multicolumn{4}{|c|}{ Neoplastic polyp (n=313) } & \multicolumn{4}{|c|}{ Advanced adenoma ( $n=249)$} \\
\hline & $\begin{array}{c}\text { Unadjusted OR } \\
\qquad(95 \% \mathrm{CI})\end{array}$ & $\mathrm{p}$-value & $\begin{array}{c}\text { Adjusted OR } \\
\text { (95\% CI) }\end{array}$ & $\mathrm{p}$-value & $\begin{array}{l}\text { Unadjusted OR } \\
\qquad(95 \% \mathrm{CI})\end{array}$ & $\mathrm{p}$-value & $\begin{array}{c}\text { Adjusted OR } \\
(95 \% \mathrm{CI})\end{array}$ & $\mathrm{p}$-value \\
\hline Sex (female) & $1.33(0.72-2.55)$ & 0.375 & $1.28(0.50-3.33)$ & 0.609 & $1.18(0.51-2.62)$ & 0.683 & $0.99(0.25-4.08)$ & 0.990 \\
\hline \multicolumn{9}{|l|}{ Age } \\
\hline $45-64 \mathrm{yr}$ & $2.70(1.12-6.34)$ & 0.024 & $2.90(0.93-9.05)$ & 0.065 & $0.35(0.09-1.71)$ & 0.146 & $0.32(0.04-3.53)$ & 0.320 \\
\hline$\geq 65 \mathrm{yr}$ & $4.18(1.63-10.70)$ & 0.003 & $2.68(0.76-9.56)$ & 0.125 & $0.78(0.22-3.70)$ & 0.724 & $0.82(0.10-9.24)$ & 0.865 \\
\hline $\operatorname{BMI}\left(\geq 23 \mathrm{~kg} / \mathrm{m}^{2}\right)$ & $0.68(0.35-1.26)$ & 0.234 & $0.66(0.28-1.48)$ & 0.327 & $0.53(0.24-1.17)$ & 0.108 & $0.68(0.19-2.43)$ & 0.548 \\
\hline Smoking & $0.66(0.30-1.40)$ & 0.287 & $0.61(0.23-1.53)$ & 0.298 & $0.65(0.20-2.04)$ & 0.453 & $0.97(0.19-4.79)$ & 0.967 \\
\hline Drinking & $1.06(0.55-2.17)$ & 0.864 & $1.38(0.57-3.47)$ & 0.477 & $0.38(0.09-1.12)$ & 0.120 & $0.29(0.04-1.55)$ & 0.168 \\
\hline $\mathrm{DM}$ & 0.93 (0.49-1.83) & 0.822 & $0.70(0.29-1.73)$ & 0.426 & $1.08(0.41-2.57)$ & 0.861 & $1.74(0.42-7.00)$ & 0.433 \\
\hline HTN & $2.07(1.15-3.90)$ & 0.019 & $2.99(1.29-7.28)$ & 0.013 & $0.90(0.41-1.95)$ & 0.798 & $0.75(0.20-2.65)$ & 0.652 \\
\hline Dyslipidemia & $0.69(0.30-1.73)$ & 0.404 & $1.39(0.42-5.09)$ & 0.597 & $0.71(0.11-2.62)$ & 0.657 & $1.06(0.11-7.34)$ & 0.954 \\
\hline Colon cancer family history & $1.19(0.30-7.91)$ & 0.830 & $0.55(0.10-4.30)$ & 0.511 & $3.94(0.80-15.89)$ & 0.062 & $0.93(0.09-7.99)$ & 0.947 \\
\hline Site (distal) & $1.02(0.58-1.76)$ & 0.952 & $0.49(0.24-1.00)$ & 0.055 & $1.45(0.66-3.36)$ & 0.367 & $0.52(0.16-1.68)$ & 0.275 \\
\hline CSM positive & $3.80(1.88-8.54)$ & 0.000 & $3.51(1.45-9.25)$ & 0.007 & $5.50(2.42-13.72)$ & 0.000 & $5.64(1.77-20.28)$ & 0.005 \\
\hline Gross (protruded) & 3.57 (1.58-9.58) & 0.005 & $4.85(1.65-17.23)$ & 0.008 & $5.34(2.43-12.14)$ & 0.000 & $3.30(1.15-9.74)$ & 0.026 \\
\hline Pit (III, IV, V) & $10.09(5.32-19.55)$ & 0.000 & $10.14(4.85-22.12)$ & 0.000 & $1.48(0.40-9.60)$ & 0.606 & $0.44(0.07-4.08)$ & 0.411 \\
\hline \multicolumn{9}{|l|}{ Size } \\
\hline $5-9 \mathrm{~mm}$ & $1.65(0.88-3.07)$ & 0.116 & $1.49(0.71-3.14)$ & 0.293 & 1.49 (0.33-10.35) & 0.632 & $1.03(0.19-8.02)$ & 0.973 \\
\hline$\geq 10 \mathrm{~mm}$ & $1.41(0.66-3.12)$ & 0.384 & $0.73(0.26-2.07)$ & 0.544 & 23.90 (6.46-155.53) & 0.000 & $18.56(3.89-147.01)$ & 0.001 \\
\hline
\end{tabular}

$\mathrm{OR}$, odds ratio; $\mathrm{Cl}$, confidence interval; BMI, body mass index; DM, diabetes mellitus; HTN, hypertension; CSM, chicken skin mucosa.

adenoma, and it may be associated with carcinogenesis rather than just a trauma response.

The CSM-positive group was divided into types 1 and 2 . It was assumed that since type 2 polyps were simply caused by mechanical damage from injection, type 2 polyps would not show statistically significant differences. However, various foamy macrophages and neoplastic features were histologically observed in type 2 CSM-positive polyps. Similar to type 1 polyps, the proportion of neoplastic polyps in type 2 was significantly different from that in CSMnegative polyps (type 0), suggesting that CSM is not a simple physical phenomenon.

Macrophages are important immune cells involved in various biological processes, such as development, metabolic regulation, and maintenance of tissue homeostasis and inflammatory response. Macrophages are essential to the progression of the pathophysiology of cancer. ${ }^{25} \mathrm{~A}$ review of macrophages involved in tumorogenesis ${ }^{26}$ revealed the potential of macrophage phenotypic regulation by physical and mechanical cues. Tissues such as the lungs, bones, and vessels have appropriate physiological thresholds for mechanical stimulation, which is important for cell growth and maturation. However, abnormal stimulation is associated with disease and inflammation. ${ }^{27,28}$ In addition, studies by Wehner et al. ${ }^{29}$ demonstrated that exposure of neonatal rat intestinal smooth muscle cells and primary peritoneal macrophages to mechanical strain increased the expression of inflammatory genes and levels of macrophage inflammatory protein (MIP)- $1 \alpha$. and MIP 2 mRNA.
It is well known that chronic inflammation is associated with tumorigenesis. Therefore, the large size, triggering abnormal inflammation and tumorigenesis of macrophages, might explain the frequent presence of CSMs around the neoplastic polyp.

In 2012, a study reported the carcinogenic risk of CSM surrounding adult colorectal adenomas. ${ }^{12}$ The expression of proliferation markers (Ki-67 and cyclooxygenase-2 [COX-2]) and apoptotic factors (survivin and caspase-3) in tissues was analyzed to determine their relationship with CSM. In this study, the levels of Ki-67 and COX-2 were significantly higher in adenomas with CSM.

The 2010 study by Wehner et al. ${ }^{29}$ demonstrated that primary rat peritoneal macrophages subjected to static stretch showed increased expression of inflammatory genes, including inducible nitric oxide synthase, COX2 , interleukin- $1 \beta$, interleukin-6, MIP- $1 \alpha$, and MIP-239. Although the study did not involve human colorectal cells, the increased expression of COX-2 in peritoneal macrophages correlated with higher levels of COX-2 in polyps around CSM, suggesting that the macrophage seen in CSM increased the expression of COX-2 via static stretch, resulting in increased expression of COX-2 in adenoma with CSM. In addition, the role of COX-2 in gut inflammation explains the relationship between CSM and neoplastic or advanced polyps via tumorigenesis induced by abnormal inflammation.

The association between CSM and polyps in the distal colon can be explained by the same mechanism. Since the 
colonic transit time is longer in the distal colon, ${ }^{30}$ abnormal macrophage activation and increased recruitment occur due to the secretion of various cytokines in the distal colon under prolonged stretch, thus explaining the greater incidence of CSM. Furthermore, as reported by Chung et al. ${ }^{13}$ the distal colon and rectum exhibit higher chances of bowel inflammation by retaining stool, bacteria, and macrophages.

In this study, eight polyps with advanced histology showed absence of CSM. There are various possibilities for this phenomenon. The abnormal inflammation or increased expression of inflammatory genes may have led to tumorigenesis despite the lack of macrophages showing CSM. Macrophages are one of the many different causes of advanced adenoma and do not explain all adenomacarcinoma sequences. Thus, polyps with advanced histology without CSM may have been observed.

However, further studies are needed to assess any differences in the number of accumulated macrophages according to the presence and absence of CSM and investigate the relationship between the absence of CSM and reduced accumulation of macrophages. Evaluation of inflammatory proteins or gene expression, in cases of absent CSM with advanced histology, would further elucidate the mechanism of CSM development.

The pit pattern is a robust parameter for differentiating between neoplastic and non-neoplastic polyps. However, it must be analyzed via chromoendoscopy rather than white light colonoscopy. In this study, the pit pattern observed via conventional colonoscopy was not related to advanced histology of polyps. It showed the limitation of pit patterns observed using conventional light colonoscopy (pit groups III, IV, and V); however, CSM was associated with advanced histology. Therefore, if the CSM is positive around the polyp, it suggests advanced histology warranting polypectomy even if the pit is not clearly identified in conventional colonoscopy.

Analysis of diagnostic performance for neoplastic pol- yps showed that sensitivity may increase from $90.73 \%$ to 93.6\% when there is at least one pit pattern or CSM. In addition, when there was no pit pattern and no CSM, the specificity for neoplastic polyps increased from $50.8 \%$ to $89.23 \%$. These findings show that simultaneous assessment of easily identifiable CSM and pit patterns during conventional light colonoscopy compensated for the limitations of pit patterns and helped diagnose neoplastic polyps.

Chung et al. ${ }^{13}$ retrospectively reported the prevalence and clinical characteristics of colorectal neoplasms with CSM using those found in the screening colonoscopy in Korea. They reported that CSM was significantly associated with advanced pathology and multiple adenomas. A prospective study on the association between CSM and neoplastic polyps were required, since polyps larger than $5 \mathrm{~mm}$ need to be removed for screening, thus simultaneously necessitating judgement regarding whether or not they are neoplastic. This decision affects the determination of whether to remove the polyp and the extent of removal. Therefore, the correlation of neoplastic polyps and CSM was prospectively examined, and when CSM was around a polyp, it was 3.51 times more likely to be malignant.

Additionally, an association between CSM (type 1) and neoplastic polyps would improve adenoma detection rate during conventional colonoscopic screening. This means that detection of CSM may lead to the diagnosis of nearby neoplastic polyps by widening the polyp boundary. Although the polyp was missed during endoscopy, observing a nearby CSM may help detect neoplastic polyps (Fig. 4).

This study had a few limitations. The findings relate to unknown underlying mechanisms of the association of CSM with neoplastic polyps, suggesting the need for biological and experimental studies investigating CSM. Furthermore, the sample size was small; however, the post-hoc analyses showed that our study had an adequate sample size at the 5\% significance level and $80 \%, 90 \%$, and $95 \%$ power, respectively. Nevertheless, the study confirmed that CSM is a significant independent variable in endoscopic

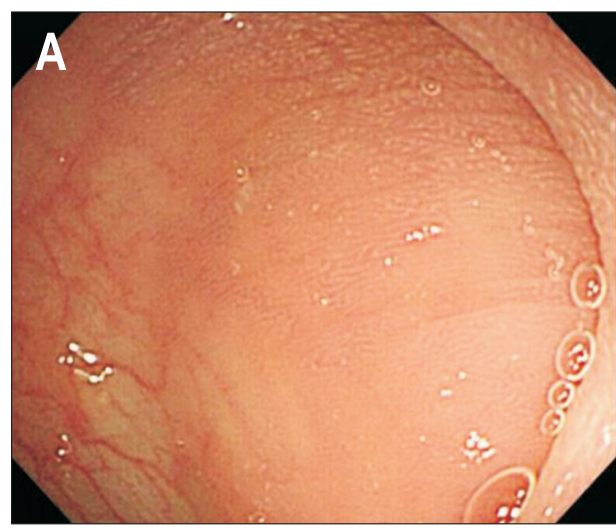

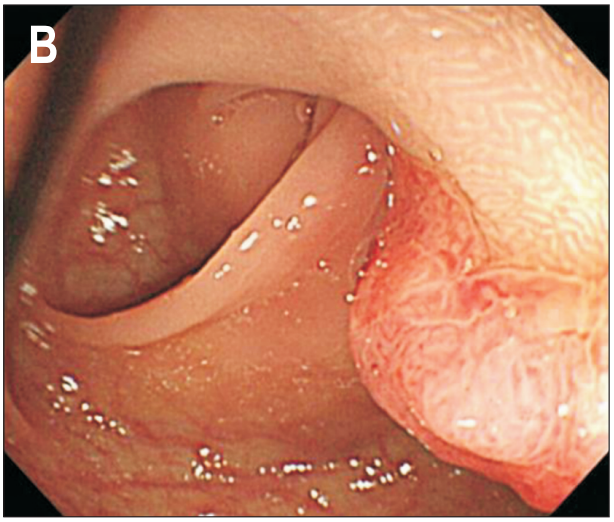

Fig. 4. A case of a polyp near chicken skin mucosa (CSM). (A) Although the polyp was missed during endoscopy, (B) the observation of a nearby CSM may help detect neoplastic polyps. 
investigations of well-differentiated and neoplastic polyps. This finding suggests that the effective pit pattern for magnifying endoscopy with NBI is limited under conventional light colonoscopy, and it is expected that CSM can be used as a complementary tool, and additional large-scale studies are needed to further support this finding. Moreover, assessment of other endoscopic findings related to advanced histology (e.g., depressed type/distorted surface pattern, color, and vessels through NBI/capillary pattern evaluation through Sano classification) to investigate the association with CSM would help to evaluate the diagnostic value.

In conclusion, neoplastic polyps, particularly advanced adenomas, were associated with positive CSM. Although the mechanism is not clear, the macrophage response to physical stimulation is important for further investigation. Our results suggest that CSM is a useful marker in differentiating neoplastic polyps and advanced polyps under conventional white colonoscopy.

\section{CONFLICTS OF INTEREST}

No potential conflict of interest relevant to this article was reported.

\section{ACKNOWLEDGEMENTS}

This study was supported by the National Research Foundation of Korea grant funded by the Korean Government (number: NRF2017R1A2B2006514).

\section{AUTHOR CONTRIBUTIONS}

Study concept and design: K.C.H. Data acquisition: Y.M.L., K.H.S., H.S.K., K.C.H., C.S.L., S.H.L. Drafting of the manuscript; critical revision of the manuscript for important intellectual content: Y.M.L., K.C.H., K.H.S. Data analysis and interpretation: Y.M.L., K.H.S., H.S.K., C.S.L., K.C.H. Statistical analysis: I.K. Obtained funding: K.C.H. Approval of final manuscript: all authors.

\section{ORCID}

Yu Mi Lee

Kyung Ho Song

Hoon Sup Koo

Choong-Sik Lee

Inseok Ko https://orcid.org/0000-0001-8096-2936

https://orcid.org/0000-0001-6661-3879

https://orcid.org/0000-0001-7303-9051

https://orcid.org/0000-0001-5725-4309

https://orcid.org/0000-0002-6348-7392
Sang Hyuk Lee https://orcid.org/0000-0003-2190-7069

Kyu Chan Huh https://orcid.org/0000-0003-3746-8419

\section{SUPPLEMENTARY MATERIALS}

Supplementary materials can be accessed at https://doi. org/10.5009/gnl210271.

\section{REFERENCES}

1. Jung KW, Won YJ, Kong HJ, Lee ES. Prediction of cancer incidence and mortality in Korea, 2019. Cancer Res Treat 2019;51:431-437.

2. Winawer SJ, Zauber AG, Ho MN, et al. Prevention of colorectal cancer by colonoscopic polypectomy. The National Polyp Study Workgroup. N Engl J Med 1993;329:19771981.

3. Zauber AG, Winawer SJ, O'Brien MJ, et al. Colonoscopic polypectomy and long-term prevention of colorectal-cancer deaths. N Engl J Med 2012;366:687-696.

4. Leslie A, Carey FA, Pratt NR, Steele RJ. The colorectal adenoma-carcinoma sequence. Br J Surg 2002;89:845-860.

5. Atia MA, Patel NC, Ratuapli SK, et al. Nonneoplastic polypectomy during screening colonoscopy: the impact on polyp detection rate, adenoma detection rate, and overall cost. Gastrointest Endosc 2015;82:370-375.

6. Inoue H, Igari T, Nishikage T, Ami K, Yoshida T, Iwai T. A novel method of virtual histopathology using laser-scanning confocal microscopy in-vitro with untreated fresh specimens from the gastrointestinal mucosa. Endoscopy 2000;32:439443.

7. Park CH, Yang DH, Kim JW, et al. Clinical practice guideline for endoscopic resection of early gastrointestinal cancer. Intest Res 2021;19:127-157.

8. Gonai T, Kawasaki K, Nakamura S, et al. Microvascular density under magnifying narrow-band imaging endoscopy in colorectal epithelial neoplasms. Intest Res 2020;18:107-114.

9. Sano Y, Ikematsu H, Fu KI, et al. Meshed capillary vessels by use of narrow-band imaging for differential diagnosis of small colorectal polyps. Gastrointest Endosc 2009;69:278283.

10. Shatz BA, Weinstock LB, Thyssen EP, Mujeeb I, DeSchryver K. Colonic chicken skin mucosa: an endoscopic and histological abnormality adjacent to colonic neoplasms. Am J Gastroenterol 1998;93:623-627.

11. Nowicki MJ, Bishop PR, Subramony C, Wyatt-Ashmead J, May W, Crawford M. Colonic chicken-skin mucosa in children with polyps is not a preneoplastic lesion. J Pediatr Gastroenterol Nutr 2005;41:600-606. 
12. Guan J, Zhao R, Zhang X, et al. Chicken skin mucosa surrounding adult colorectal adenomas is a risk factor for carcinogenesis. Am J Clin Oncol 2012;35:527-532.

13. Chung EJ, Lee JY, Choe J, et al. Colonic chicken skin mucosa is an independent endoscopic predictor of advanced colorectal adenoma. Intest Res 2015;13:318-325.

14. Lee YM, Song KH, Song HJ, et al. Do whitish spots around colorectal polyp give additional information under conventional colonoscopy? Paper presented at: 62nd Congress of the Korean Society of Gastrointestinal Endoscopy; 2012 Nov 24; Seoul, Korea.

15. Schlemper RJ, Riddell RH, Kato Y, et al. The Vienna classification of gastrointestinal epithelial neoplasia. Gut 2000;47: 251-255.

16. National Institutes of Health (NIH) National Cancer Institute. Dictionary of cancer terms: proximal colon [Internet]. Bethesda: NIH [cited 2021 Aug 5]. Available from: http:// www.cancer.gov/publications/dictionaries/cancer-terms/def/ proximal-colon.

17. National Institutes of Health (NIH) National Cancer Institute. Dictionary of cancer terms: distal colon [Internet]. Bethesda: NIH [cited 2021 Aug 5]. Available from: https:// www.cancer.gov/publications/dictionaries/cancer-terms/def/ distal-colon.

18. Ramnani DM. Neoplastic polyps [Internet]. Richmond: Webpathology; c2018 [cited 2021 Aug 5]. Available from: http://www.webpathology.com/case.asp?case $=221$.

19. Levin B, Lieberman DA, McFarland B, et al. Screening and surveillance for the early detection of colorectal cancer and adenomatous polyps, 2008: a joint guideline from the American Cancer Society, the US Multi-Society Task Force on Colorectal Cancer, and the American College of Radiology. Gastroenterology 2008;134:1570-1595.

20. Vleugels JLA, Hazewinkel Y, Dekker E. Morphological classifications of gastrointestinal lesions. Best Pract Res Clin Gastroenterol 2017;31:359-367.
21. Kudo S, Tamura S, Nakajima T, Yamano H, Kusaka H, Watanabe $\mathrm{H}$. Diagnosis of colorectal tumorous lesions by magnifying endoscopy. Gastrointest Endosc 1996;44:8-14.

22. Faul F, Erdfelder E, Lang AG, Buchner A. G*Power 3: a flexible statistical power analysis program for the social, behavioral, and biomedical sciences. Behav Res Methods 2007;39: 175-191.

23. El-Hodhod MA, Soliman AA, Hamdy AM, Abdel-Rahim AA, Abdel-Hamid FK. Fate and ultra-structural features of chicken skin mucosa around juvenile polyps. Acta Gastroenterol Belg 2011;74:17-21.

24. Nowicki MJ, Subramony C, Bishop PR, Parker PH. Colonic chicken skin mucosa: association with juvenile polyps in children. Am J Gastroenterol 2001;96:788-792.

25. Yang M, McKay D, Pollard JW, Lewis CE. Diverse functions of macrophages in different tumor microenvironments. Cancer Res 2018;78:5492-5503.

26. McWhorter FY, Davis CT, Liu WF. Physical and mechanical regulation of macrophage phenotype and function. Cell Mol Life Sci 2015;72:1303-1316.

27. Dos Santos CC, Slutsky AS. Invited review: mechanisms of ventilator-induced lung injury: a perspective. J Appl Physiol (1985) 2000;89:1645-1655.

28. Mourgeon E, Isowa N, Keshavjee S, Zhang X, Slutsky AS, Liu M. Mechanical stretch stimulates macrophage inflammatory protein-2 secretion from fetal rat lung cells. Am J Physiol Lung Cell Mol Physiol 2000;279:L699-L706.

29. Wehner S, Buchholz BM, Schuchtrup S, et al. Mechanical strain and TLR4 synergistically induce cell-specific inflammatory gene expression in intestinal smooth muscle cells and peritoneal macrophages. Am J Physiol Gastrointest Liver Physiol 2010;299:G1187-G1197.

30. Wagener S, Shankar KR, Turnock RR, Lamont GL, Baillie CT. Colonic transit time: what is normal? J Pediatr Surg 2004;39:166-169. 